\title{
Poly(2-alkyl-2-oxazoline) electrode interlayers for improved n-type organic field effect transistor performance
}

\author{
Sungho Nam, ${ }^{1}$ Victor R. De la Rosa, ${ }^{2}$ Yuljae Cho, ${ }^{3}$ Rick Hamilton, ${ }^{1}$ SeungNam \\ Cha, ${ }^{3,4}$ Richard Hoogenboom, ${ }^{2}$ and Donal D. C. Bradley ${ }^{1,3,5 a)}$
}

${ }^{1}$ Department of Physics, Division of Mathematical, Physical and Life Sciences, University of Oxford, Oxford OX1 3PU,

United Kingdom

${ }^{2}$ Supramolecular Chemistry Group, Centre of Macromolecular Chemistry (CMaC), Department of Organic and Macromolecular Chemistry, Ghent University, Krijgslaan 281 S4, B-9000, Ghent, Belgium.

${ }^{3}$ Department of Engineering Science, Division of Mathematical, Physical and Life Sciences, University of Oxford, Oxford OX1 3PJ, United Kingdom

${ }^{4}$ Department of Physics, Sungkyunkwan University, Suwon, Gyeonggi-do, 16419, Republic of Korea

${ }^{5}$ Physical Science and Engineering Division, King Abdullah University of Science and Technology, Thuwal 23955-6900, KSA

\begin{abstract}
Thin film interlayer materials inserted at the metal/semiconductor interface provide an effective means to improve charge injection and reduce threshold voltage for organic field-effect transistors. Here we report the use of poly(2-alkyl-2-oxazoline) interlayers for gold electrodes within n-type poly[[ $N, N^{\prime}$-bis(2-octyldodecyl)-naphthalene-1,4,5,8-bis(dicarboximide)-2,6-diyl]alt-5,5'-(2,2'-bithiophene)] field-effect transistors. We specifically show that the use of poly(2-ethyl-2-oxazoline) yields a reduction in work function from 5.07 to $4.73 \mathrm{eV}(\Delta \mathrm{E}=0.34 \mathrm{eV})$, an increase in electron mobility from 0.04 to $0.15 \mathrm{~cm}^{2} / \mathrm{Vs}$ (3.75 times) and a reduction in threshold voltage from 27.5 to $16.5 \mathrm{~V}(\Delta \mathrm{V}=11 \mathrm{~V})$ relative to bare gold. The alkyl side chain of the poly(2-alkyl-2-oxazoline)s has a significant influence on film microstructure and, as a consequence, also device performance.
\end{abstract}

\section{THE MANUSCRIPT}

Organic field effect transistors (OFETs) are of interest for applications including rollable and foldable information displays and sensors for use in the internet of things (IoT) ${ }^{1-5}$ Significant progress in the performance of OFETs has been achieved through fundamental understanding of the device physics and charge transport for organic semiconductors as well as through the rational design and synthesis of new materials. ${ }^{6-9}$ Many of the applications envisioned for OFETs are portable electronic devices for which low power consumption is a critical requirement. ${ }^{10,11}$ Complementary logic, combining p-type and n-type OFETs, can help to minimize power consumption. However, the performance of n-type OFETs typically lags behind in terms of charge carrier mobility and air stability. ${ }^{12}$ High-performance n-type OFETs are, therefore, a key need for the on-going development of plastic electronics and provide the focus for this paper.

a)Electronic mail: Donal.Bradley@kaust.edu.sa 
An effective approach to improving the performance of n-type OFETs is to better control electron injection from the metal electrode into the lowest unoccupied molecular orbital (LUMO) of the organic semiconductor. In general, low work function metals, such as $\mathrm{Ca}, \mathrm{Mg}$ and $\mathrm{Al}$ (with work function $\Phi \approx 2.8,3.6$ and $4.2 \mathrm{eV}$ respectively), reduce the energy barrier for electron injection into organic semiconductors. ${ }^{13}$ However, these metals are prone to ambient oxidation, producing stable but insulating oxide layers for $\mathrm{Al}$ and $\mathrm{Mg}$ but resulting in destruction of the electrode for $\mathrm{Ca}$; in both situations this leads to rapid decay of OFET performance. ${ }^{14} \mathrm{~A}$ key strategy in the quest for air-stable and high-performance n-type OFETs has therefore been to interface-engineer higher work function electrodes like $\mathrm{Au}(\Phi \approx 5.1 \mathrm{eV})$ that are impervious to ambient oxidation. Extensive studies have been undertaken on work-function-lowering interlayer materials for this purpose, including their use for organic semiconductor transistors, electronic and light emitting diodes, solar cells and photodetectors. These include water and alcoholsoluble conjugated and non-conjugated polymers (both neutral dipolar and ionic (polyelectrolyte) versions), alkali metal salts, self-assembled dipolar monolayers, metal oxide and carbonate thin films. ${ }^{15-25}$

In particular, non-conjugated neutral dipolar polymers have shown good air stability as well as having the desired effect on energy barriers, leading to improved performance for organic light-emitting diodes, organic solar cells and OFETs. ${ }^{14}$ In this vein, we recently reported the use of poly(2-ethyl-2-oxazoline) (PEtOx) as an effective interlayer for inverted polymer solar cells, yielding $\geq 10 \%$ efficiency for a nano-dot interlayer microstructure. ${ }^{26}$ Subsequent studies have incorporated a similar PEtOx work function lowering nanodot layer in silver-contacted perovskite solar cells ${ }^{27,28}$ and mixed PEtOx into perovskite thin films to enhance LED operation. ${ }^{29}$ We have further shown the suitability of $\mathrm{ZnO} / \mathrm{PEtOx}$ composite fims with a nanocrater morphology to enhance electron collection efficiency and stability in polymer:non-fullerene solar cells. ${ }^{32}$ These studies were all limited to the use of PEtOx and other poly(2-alkyl-2-oxazoline)s (PAOx) have not yet been explored for these types of applications.

Here we extend the use of PAOx as an electron-injecting/hole-blocking interlayer for Au source-drain electrode n-type OFETs.

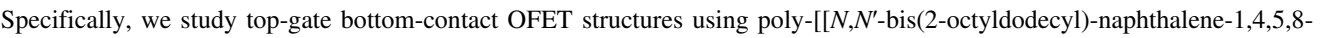
bis(dicarboximide)-2,6-diyl]-alt-5,5'-(2,2'-bithiophene)] (P(NDI2OD-T2) as semiconductor with polymethylmethacrylate (PMMA) as gate insultator. We examine the effect of PAOx alkyl side chain length on OFET performance, comparing the observed behavior with changes in the work function of PAOx-coated Au electrodes and PAOx thin film microstructures. Whilst the choice of alkyl side chain in PAOx is known to significantly affect a number of physico-chemical properties, ${ }^{30-33}$ 
such as glass transition temperature ( $\mathrm{Tg}$ ), crystallinity and surface energy (and its dependence on annealing), very little is known to date about its effect on device performance.

\section{Figure 1}

A set of hydroxyl-terminated PAOx samples (see Figure 1(a) for chemical structures) was synthesized, as described previously, via cationic ring-opening polymerization of 2-alkyl substituted 2-oxazolines with alkyl side chain length varied from methyl (poly(2-methyl-2-oxazoline) (PMeOx)) to ethyl (PEtOx) to propyl (poly(2-n-propyl-2-oxazoline) (PPrOx)). The resulting weight-average molecular weights (Mw) were 12.3, 12.7 and $11.6 \mathrm{kDa}$ with dispersities $(\bigoplus)$ of $1.08,1.10$ and 1.09, respectively. PMMA (Mw = $120 \mathrm{kDa}$; $=2.2$; Figure 1(b)) and $\mathrm{P}(\mathrm{NDI} 2 \mathrm{OD}-\mathrm{T} 2)(\mathrm{Mw}=25-50 \mathrm{kDa} ; \mathrm{Ð}=1.5-3.5$; Figure 1(b)) were purchased from Sigma Aldrich (United States) and 1-Material (Canada), respectively, and used as received. PMMA was selected as a low- $k(k=3.5)$ gate dielectric for our study on the basis of its chemical stability and low surface energy, minimizing interfacial/bulk charge trap densities. ${ }^{30} \mathrm{P}(\mathrm{NDI} 2 \mathrm{OD}-\mathrm{T} 2)$ is a naphthalenediimide-based semiconducting polymer with highest occupied (HOMO) and lowest unoccupied (LUMO) molecular orbital levels at -5.9 and $-4.4 \mathrm{eV}$, respectively, that has been used previously to fabricate OFETs and as an electron acceptor in all-polymer solar cells. ${ }^{30,31}$

PAOx/methanol, PMMA/n-butyl acetate and P(NDI2OD-T2)/chlorobenzene solutions were prepared at a concentration of 2, 80 and $5 \mathrm{mg} / \mathrm{ml}$, respectively, and were vigorously mixed using a magnetic stirrer at room temperature for 24 hours before spin-coating. Glass substrates were cleaned in an ultrasonic bath, first with acetone then isopropyl alcohol for $10 \mathrm{~min}$ each, and dried under flowing nitrogen gas. 30-nm-thickness Au source/drain (S/D) electrodes (channel width 1 mm and length 80 $\mu \mathrm{m})$ were vacuum-evaporated $\left(5 \times 10^{-6}\right.$ Torr $)$ onto the glass substrates through a shadow mask. PAOx films were spin-coated (4000 rpm, $30 \mathrm{~s}$ ) on top, followed by thermal annealing $\left(120^{\circ} \mathrm{C}, 10 \mathrm{~min}\right)$; the same procedure was followed for all three PAOx (Figure 1(a)). Next P(NDI2OD-T2) was spin-coated $(2000 \mathrm{rpm}, 40 \mathrm{~s})$ on top of the PAOx and annealed $\left(120{ }^{\circ} \mathrm{C}, 20 \mathrm{~min}\right.$ in a dry box with relative humidity $<15 \%)$. Then 600 -nm-thickness films of PMMA were spin-coated $(1500 \mathrm{rpm}, 60 \mathrm{~s})$ as a gate dielectric $\left(\mathrm{C}_{\mathrm{i}}=5.5 \mathrm{nF} / \mathrm{cm}^{2}\right)$ onto the $\mathrm{P}(\mathrm{NDI} 2 \mathrm{OD}-\mathrm{T} 2)$ and soft-baked $\left(70{ }^{\circ} \mathrm{C}, 20 \mathrm{~min}\right)$. Finally, $50 \mathrm{~nm}$-thickness silver (Ag) gate electrodes were vacuum-evaporated $\left(5 \times 10^{-6}\right.$ Torr) on top (see schematic in Figure $\left.1(\mathrm{~b})\right)$. Film thicknesses were measured using a surface profilometer (Veeco Dektak 150) and OFET characteristics using a semiconductor parameter analyzer (4200SCS, Keithley) connected to a probe station (Cascade Microtech), all under ambient conditions. The work functions of the 
PAOx films were determined under ambient conditions using a Kelvin probe (KP Technology) measurement system with a gold disc tip ( $2 \mathrm{~mm}$ diameter), calibrated against highly oriented pyrolytic graphite, and their surface microstructures were probed using an atomic force microscope (AFM, Veeco Dimension 3100).

\section{Figure 2}

As shown in Fig. 2a, the transfer characteristics of P(NDI2OD-T2) OFETs were significantly influenced by insertion of the PAOx interlayer. In particular, at moderate drain-source voltages (see left panel for $V_{D S}=20 \mathrm{~V}$ ), devices with PAOx interlayers exhibited higher n-channel drain current $\left(I_{D S}\right)$, especially at higher $V_{G S}$ values, and lower turn-on voltages $\left(V_{O N}\right)$ than devices with bare gold. The effect was particularly pronounced for the PEtOx interlayer with a more than one order of magnitude increase in $I_{D S}$ at $V_{G S}=80 \mathrm{~V}$ from $0.86 \mu \mathrm{A}$ to $20.70 \mu \mathrm{A}$ and a decrease in $V_{O N}$ from $15 \mathrm{~V}$ for bare gold to $4 \mathrm{~V}$. At high drainsource voltages (see right panel for $V_{D S}=80 \mathrm{~V}$ ), ambipolar operation is observed for all devices with a significant p-channel current becoming evident ${ }^{18}$ For bare gold the two channels are relatively symmetrical but upon insertion of a PAOx interlayer the n-channel becomes more dominant. Again, this is particularly the case for PEtOx, for which there is a simultaneous overall increase in n-channel and reduction in p-channel current and also a reduction in $V_{O N}$. For PMeOx and PPrOx interlayers, however, the main effect is a reduction in p-channel current, with PMeOx being most effective in that regard; the n-channel currents at $V_{G S}=80 \mathrm{~V}$ are the same as for bare gold.

The output characteristics for P(NDI2OD-T2) OFETs with (right panel) and without (left panel) a PEtOx interlayer are shown in Fig. 2b; results for PMeOx and PPrOx interlayers show qualitatively similar but much less pronounced changes. At $V_{D S}=$ $V_{G S}=80 \mathrm{~V}$, the PEtOx interlayer device gives $I_{D S}=21.6 \mu \mathrm{A}$, some three times higher than for the interlayer-free device where $I_{D S}=7.03 \mu \mathrm{A}$. The improved performance for $\mathrm{Au} / \mathrm{PEtOx}$ over bare Au can be attributed to a reduced contact resistance, as will be discussed further below.

Figure 3 
The threshold voltages $\left(V_{T H}\right)$ and field-effect mobilities in the saturation region $\left(\mu_{s a t}\right)$, for devices without and with PAOx interlayers, were obtained from a plot of the square root of $I_{D S} v s V_{G S}$ at $\mathrm{V}_{\mathrm{DS}}=80 \mathrm{~V}$ (see Fig. $3 \mathrm{a}$ and b). The $V_{T H}$ value (inset Fig. 3a) for devices with a PEtOx interlayer $(16.5 \mathrm{~V})$ is much lower than for bare gold devices $(27.5 \mathrm{~V})$, whereas the devices with PMeOx (39.1 V) and PPrOx (37.4 V) exhibited higher $V_{T H}$ values. In respect of $\mu_{s a t}$, all three PAOx interlayer devices exhibited higher mobility than the device without an interlayer, yielding $\mu_{\text {sat }}=0.07$ (PMeOx), 0.15 (PEtOx) and 0.06 (PPrOx), versus $0.04 \mathrm{~cm}^{2} \mathrm{~V}^{-1} \mathrm{~s}^{-1}$ for bare $\mathrm{Au}$. In particular, the PEtOx interlayer gives a 3.75 times higher $\mu_{\text {sat }}$ value. In addition, the trend of the field-effect mobilities in the linear region $\left(\mu_{\text {lin }}\right)$ (see inset to Fig. 3b) is consistent with that of $\mu_{\text {sat. }}$ Such enhanced performance is expected to correlate inversely with the contact resistance, $R_{C}$, times channel width, $W$, product $R_{C} W$ (Fig. $3 \mathrm{~d}$ ) which was calculated using the transfer length method by extrapolating the total resistance times channel width product, $R_{\text {total }} W$ (Fig. $3 \mathrm{c}$ ), to zero channel length. ${ }^{22}$ The decreased $V_{T H}$ and increased $\mu_{\text {sat }}$ values for PEtOx interlayer devices are then consistent with the observed reduction in $R c W$ to $0.54 \times 10^{7} \Omega \mathrm{cm}$ (see Fig. 3d), compared to $4.58 \times 10^{7} \Omega \mathrm{cm}$ for bare Au. For PMeOx and PPrOx interlayers we find $R c W=5.88$ and $5.75 \times 10^{7} \Omega \mathrm{cm}$, respectively, both higher than for bare gold and, therefore, consistent with their higher $V_{T H}$ values. In spite of this, their $\mu_{\text {sat }}$ values are slightly higher than for bare Au (see Fig. 3b). Finding ways to reduce the contact resistance for PMeOx and PPrOx should allow better results for these two polymers and would be a useful future direction for study. We note, however, that similar values have been previously reported for n-type naphthalene diimide (NDI)-based polymer transistors. ${ }^{32-35}$

Figure 4

Kelvin probe measurements were used to study the changes in electrode work function in the presence of PAOx interlayers; Fig. 4a presents the results. The bare Au work function was measured to be $\Phi \approx 5.07 \mathrm{eV}$, with $\Phi \approx 4.75,4.73$, and $4.74 \mathrm{eV}$ for $\mathrm{Au} / \mathrm{PMeOx}, \mathrm{Au} / \mathrm{PEtOx}$, and $\mathrm{Au} / \mathrm{PPrOx}$, respectively. The large energy barrier $(\approx 0.67 \mathrm{eV})$ between the work function of bare $\mathrm{Au}$ and the LUMO energy level of P(NDI2OD-T2) hampers electron injection. All three PAOx dipole layers reduce this barrier (to $0.34 \pm 0.1 \mathrm{eV}$ ), suggesting that the subsequent difference in OFET parameters must have a different origin. It is also not obvious why $V_{T H}$ should be higher for $\mathrm{Au} / \mathrm{PMeOx}$ and $\mathrm{Au} / \mathrm{PPrOx}$ than bare $\mathrm{Au}$. Another factor to consider is the microstructure of the PAOx interlayer films. AFM measurements on films spin-coated on glass substrates reveal, as shown in Fig. $4 \mathrm{~b}$, that the PEtOx film has a relatively smooth surface with a root-mean-square roughness $\left(R_{R M S}\right)$ of only $0.17 \mathrm{~nm}$. In comparison the PMeOx and PPrOx nanolayers are significantly rougher, with $R_{R M S}=0.28$ and $0.65 \mathrm{~nm}$, respectively. Rough films of this type 
are expected to have a fluctuating dipole orientation that can act as a scattering field for charge carriers within the channel, plausibly explaining (at least in part) the lower $\mu_{\text {sat }}$ values that are observed. Thicker films may also contribute to a higher contact resistance.

In conclusion, we have demonstrated that neutral dipolar polymers in the PAOx family can be used to improve the properties of P(NDI2OD-T2)-based n-type OFETs with Au S/D electrodes. In particular, devices with a PEtOx interlayer show a large increase in $\mu_{\text {sat }}$ to $0.15 \mathrm{~cm}^{2} / \mathrm{Vs}\left(v s 0.04 \mathrm{~cm}^{2} / \mathrm{Vs}\right.$ for bare $\mathrm{Au}$ ), with additionally a considerable decrease in $V_{T H}$ to $16.5 \mathrm{~V}$ ( $v s 27.5$ V for bare Au). Kelvin probe measurements show that the PAOx interlayers consistently reduce the work function of Au by some 0.32 to $0.34 \mathrm{eV}$, providing a pseudo-ohmic contact to $\mathrm{P}(\mathrm{NDI} 2 \mathrm{OD}-\mathrm{T} 2)$. The consequent improvement in OFET characteristics is not, however, uniform. In all three cases there is an increase in $\mu_{\text {sat }}$ but for PMeOx and PPrOx $V_{T H}$ is also increased. A contributory factor appears to be the RMS-roughness of the deposited PAOx layers that has the potential to create a fluctuating dipole field that will scatter charge carriers. Further work will be undertaken to improve the deposition processes for these two materials in order to check this hypothesis. In addition, PEtOx is a precursor material for the preparation of poly(ethylene imine) (PEI), which has also been used as an interlayer for organic electronics. ${ }^{15}$ A direct comparison of PEI and PEtOx performance would also be of interest.

\section{ACKNOWLEDGMENTS}

DDCB thanks the University of Oxford for start-up funds including a research fellowship for SN.

\section{REFERENCES}

$1 \quad$ K. Fukuda and T. Someya, Adv. Mater. 29, 1602736 (2017).

2 E. Orgiu and P. Samori, Adv. Mater. 26, 1827 (2014).

3 P. Heremans, A.K. Tripathi, A. de Jamblinne de Meux, E.C. Smits, B. Hou, G. Pourtois, and G.H. Gelinck, Adv. Mater. 28, 4266 (2016).

$4 \quad$ Y. Zang, D. Huang, C.A. Di, and D. Zhu, Adv. Mater. 28, 4549 (2016).

5 B. Kang, W.H. Lee, and K. Cho, ACS Appl. Mater. Interfaces 5, 2302 (2013)

6 H. Sirringhaus, Adv. Mater. 26, 1319 (2014).

7 D. Natali and M. Caironi, Adv. Mater. 24, 1357 (2012) 
8 C. Wang, H. Dong, W. Hu, Y. Liu, and D. Zhu, Chem Rev 112, 2208 (2012).

9 S. Holliday, J.E. Donaghey, and I. McCulloch, Chem. Mater. 26, 647 (2014).

10 J. Mei, Y. Diao, A.L. Appleton, L. Fang, and Z. Bao, J. Am. Chem. Soc. 135, 6724 (2013).

11 G. Dell'Erba, A. Luzio, D. Natali, J. Kim, D. Khim, D.-Y. Kim, Y.-Y. Noh, and M. Caironi, Appl. Phys. Lett. 104, 153303 (2014).

12 Y. Zhao, Y. Guo, and Y. Liu, Adv. Mater. 25, 5372 (2013).

13 L.-L. Chua, Z. Jana, J.-F. Chang, E.C.-W. Ou, P.K.-H. Ho, H. Sirringhaus, and R.H. Friend, Nature 434, 192 (2005).

14 T. Chiba, Y.-J. Pu, and J. Kido, J. Mater. Chem. C 3, 11567 (2015).

15 Y. Zhou, C. Fuentes-Hernandez, J. Shim, J. Meyer, A.J. Giordano, H. Li, P. Winget, T. Papadopoulos, H. Cheun, J. Kim, M. Fenoll, A. Dindar, W. Haske, E. Najafabadi, T.M. Khan, H. Sojoudi, S. Barlow, S. Graham, J.-L. Brédas, S.R. Marder, A. Kahn, and B. Kippelen, Science 327 (2012).

16 B. Sun, W. Hong, E.S. Thibau, H. Aziz, Z.H. Lu, and Y. Li, ACS Appl. Mater. Interfaces 7, 18662 (2015).

17 Y.J. Park, M.J. Cha, Y.J. Yoon, S. Cho, J.Y. Kim, J.H. Seo, and B. Walker, Adv. Electron. Mater., 1700184 (2017).

18 D. Khim, K.-J. Baeg, J. Kim, J.-S. Yeo, M. Kang, P.S.K. Amegadzea, M.-G. Kim, J. Cho, J.H. Lee, D.-Y. Kim, and Y.-Y. Noh, J. Mater. Chem. 22, 16979 (2012).

19 K. Gunasekar, W. Cho, D.X. Long, S.S. Reddy, M. Song, Y.-Y. Noh, and S.-H. Jin, Adv. Electron. Mater. 2, 1600086 (2016).

20 J. Kim, D. Khim, R. Kang, S.H. Lee, K.J. Baeg, M. Kang, Y.Y. Noh, and D.Y. Kim, ACS Appl. Mater. Interfaces 6, 8108 (2014)

21 N. Chakravarthi, K. Gunasekar, W. Cho, D.X. Long, Y.-H. Kim, C.E. Song, J.-C. Lee, A. Facchetti, M. Song, Y.-Y. Noh, and S.-H. Jin, Energy Environ. Sci. 9, 2595 (2016).

22 C. Liu, Y. Xu, and Y.-Y. Noh, Materials Today 18, 79 (2015).

23 N.K. Kim, D. Khim, Y. Xu, S.H. Lee, M. Kang, J. Kim, A. Facchetti, Y.Y. Noh, and D.Y. Kim, ACS Appl. Mater. Interfaces 6, 9614 (2014).

24 M. Suh, J. Bailey, S.W. Kim, K. Kim, D.J. Yun, Y. Jung, I. Hamilton, N. Chander, X. Wang, D.D.C. Bradley, D.Y. Jeon, and J.S. Kim, ACS Appl. Mater, Interfaces 7, 26566 (2015).

25 S. Nam, J. Seo, H. Han, H. Kim, S.G. Hahm, M. Ree, Y.-S. Gal, T.D. Anthopoulos, D.D.C. Bradley, and Y. Kim, Adv. Mater. Interfaces 3 (2016).

26 S. Nam, J. Seo, S. Woo, W.H. Kim, H. Kim, D.D. Bradley, and Y. Kim, Nat. Commun. 6, 8929 (2015). 
27 W. Chen, Y. Zhu, Y. Yu, L. Xu, G. Zhang, and Z. He, Chem. Mater. 28, 4879 (2016).

28 W. Chen, G.-N. Zhang, L.-M. Xu, R. Gu, Z.-H. Xu, H.-J. Wang, and Z.-B. He, Mater. Today Energy 1-2, 1 (2016).

29 H. Lin, L. Zhu, H. Huang, C.J. Reckmeier, C. Liang, A.L. Rogach, and W.C. Choy, Nanoscale 8, 19846 (2016).

30 S. Nam, J. Seo, H. Han, H. Kim, D.D.C. Bradley, and Y. Kim, ACS Appl. Mater. Interfaces 9, 14983 (2017).

31 S. Shi, J. Yuan, G. Ding, M. Ford, K. Lu, G. Shi, J. Sun, X. Ling, Y. Li, and W. Ma, Adv. Funct. Mater. 26, 5669 (2016).

32 Y. An, D.X. Long, Y. Kim, Y.Y. Noh, and C. Yang, Phys. Chem. Chem. Phys. 18, 12486 (2016).

33 Y. Kim, D.X. Long, J. Lee, G. Kim, T.J. Shin, K.-W. Nam, Y.-Y. Noh, and C. Yang, Macromolecules 48, 5179 (2015).

34 K. Gunasekar, W. Cho, D.X. Long, S.S. Reddy, M. Song, Y.-Y. Noh, and S.-H. Jin, Adv. Electron. Mater. 2 (2016).

35 J. Roh, T. Lee, C.M. Kang, J. Kwak, P. Lang, G. Horowitz, H. Kim, and C. Lee, Sci. Rep. 7, 46365 (2017). 
(a)

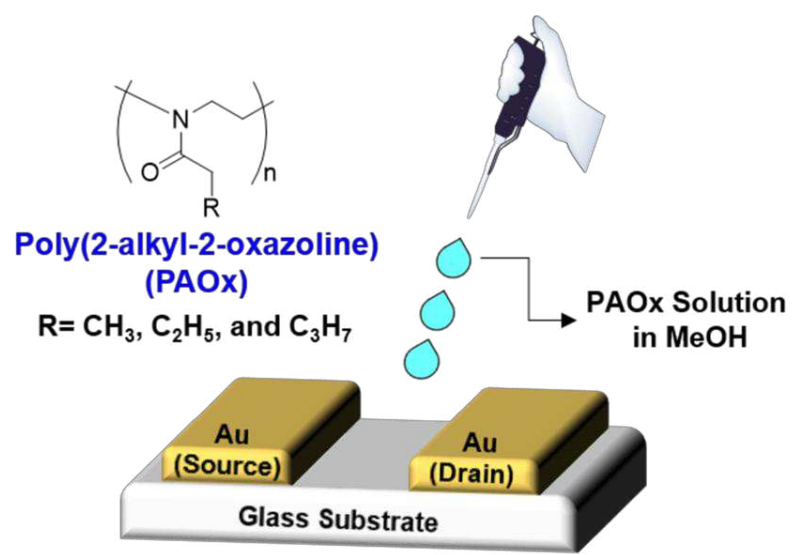

(b)

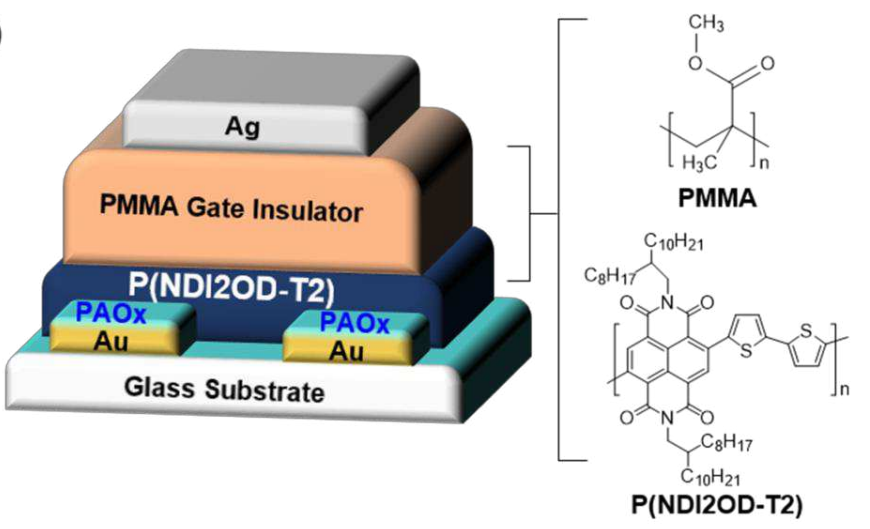

FIG. 1 (a) Chemical structure for PAOx and schematic of glass substrate with source and drain Au electrodes. (b) Schematic device structure for top-gate and bottom-contact (TG/BC) OFETs, together with chemical structures of P(NDI2OD-T2) and PMMA. 

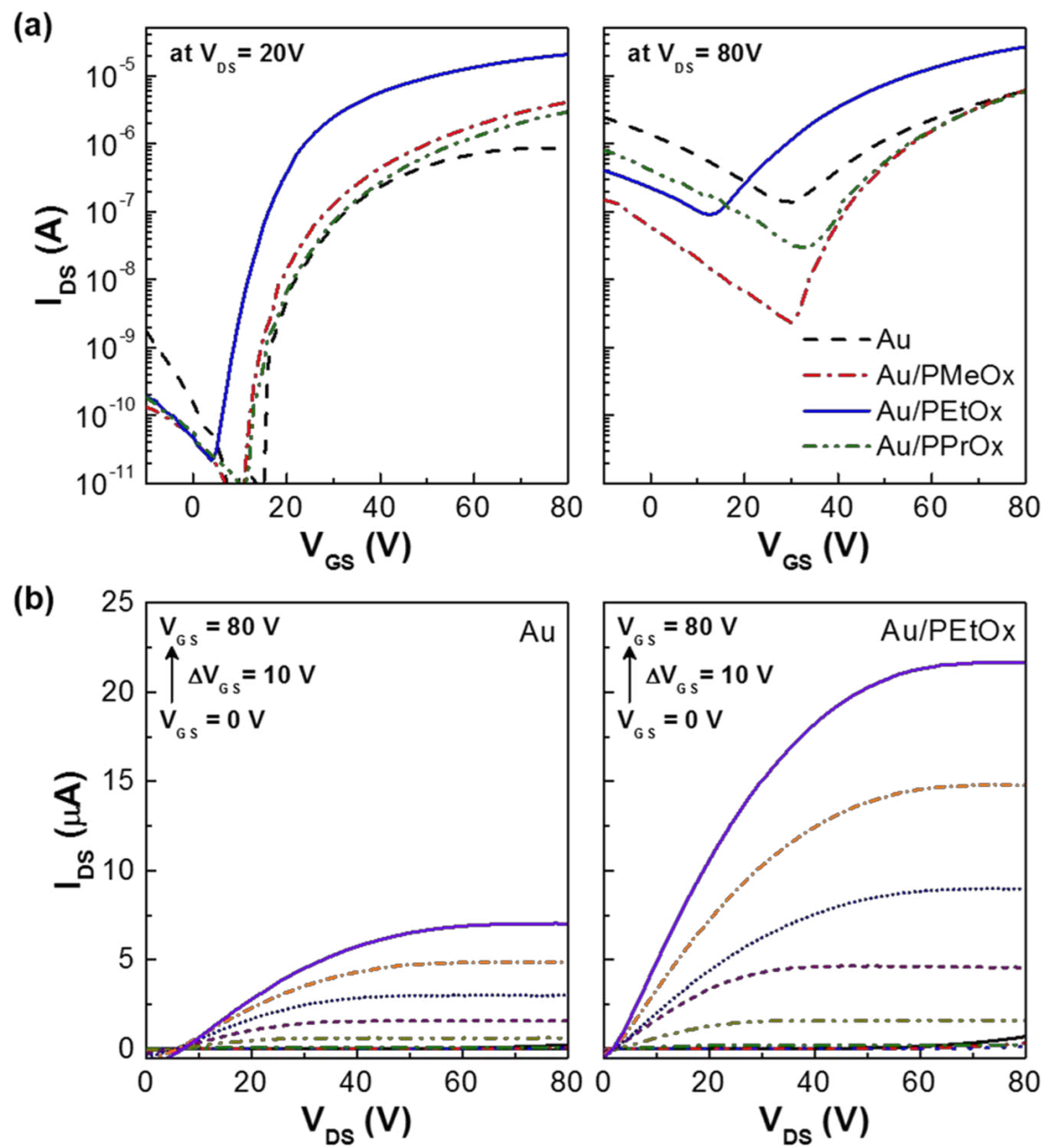

FIG. 2 (a) Transfer characteristics for P(NDI2OD-T2) OFETs without and with PMeOx, PEtOx, and PPrOx interlayers at $V_{D S}$ $=20$ and $80 \mathrm{~V}$. (b) Output characteristics for the P(NDI2OD-T2) OFETs without and with PEtOx interlayers. 

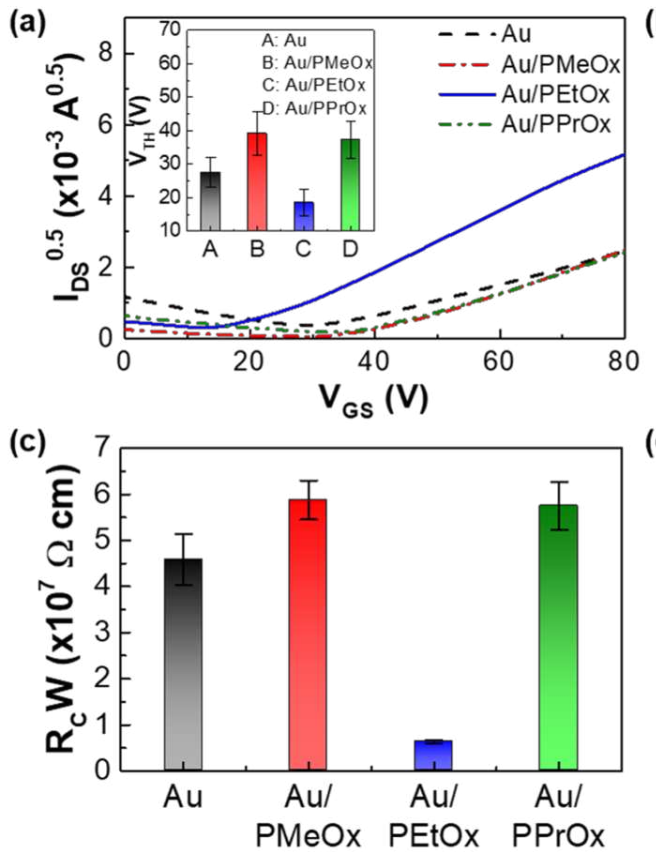

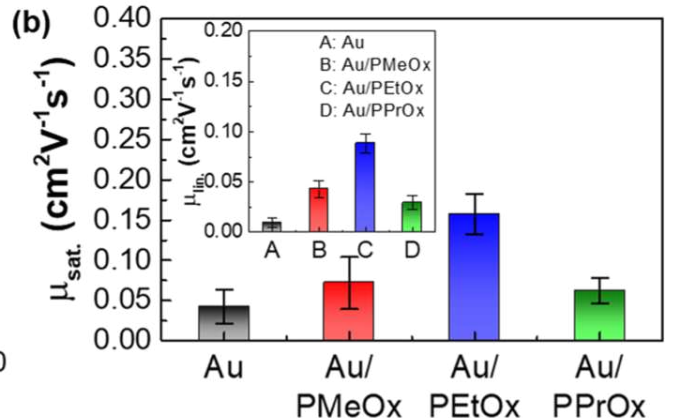

(d)

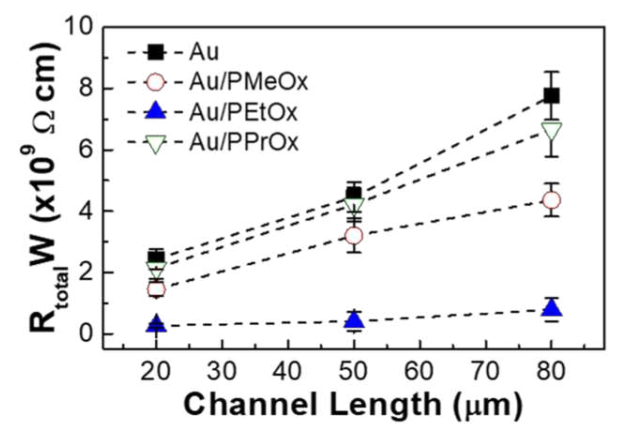

FIG. 3 (a) Square root of drain current $\left(I_{D S}{ }^{0.5}\right) v s$ gate voltage $\left(V_{G S}\right)$ for P(NDI2OD-T2) OFETs without and with PAOx interlayers. The inset shows the deduced device threshold voltage values $\left(V_{T H}\right)$. (b) Electron mobility in the saturation region $\left(\mu_{s a t}\right)$ for P(NDI2OD-T2) OFETs without and with PAOx interlayers. The inset shows the electron mobility in the linear region $\left(\mu_{\text {lin }}\right)$. (c) Deduced contact resistivity $\left(R_{C} W\right)$, determined by the transfer length method and (d) Total resistivity $\left(R_{\text {total }} W\right)$ as a function of channel length. Note that all transistor parameters were extracted from more than 10 devices and the averaged values are displayed with corresponding standard deviation. 


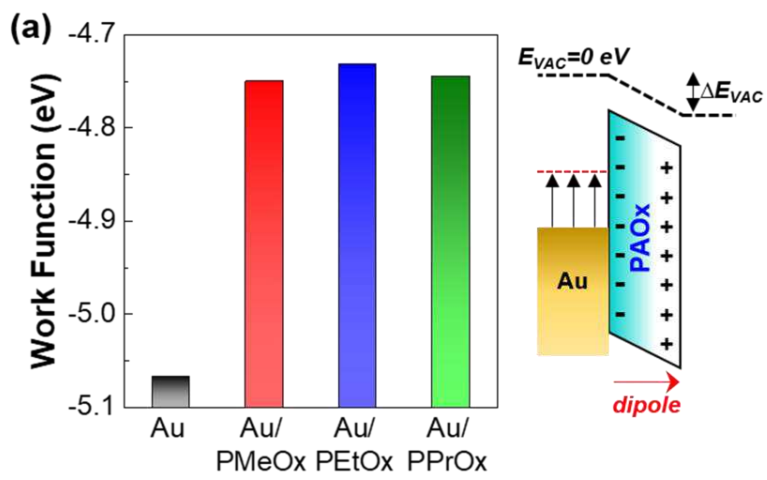

(b)
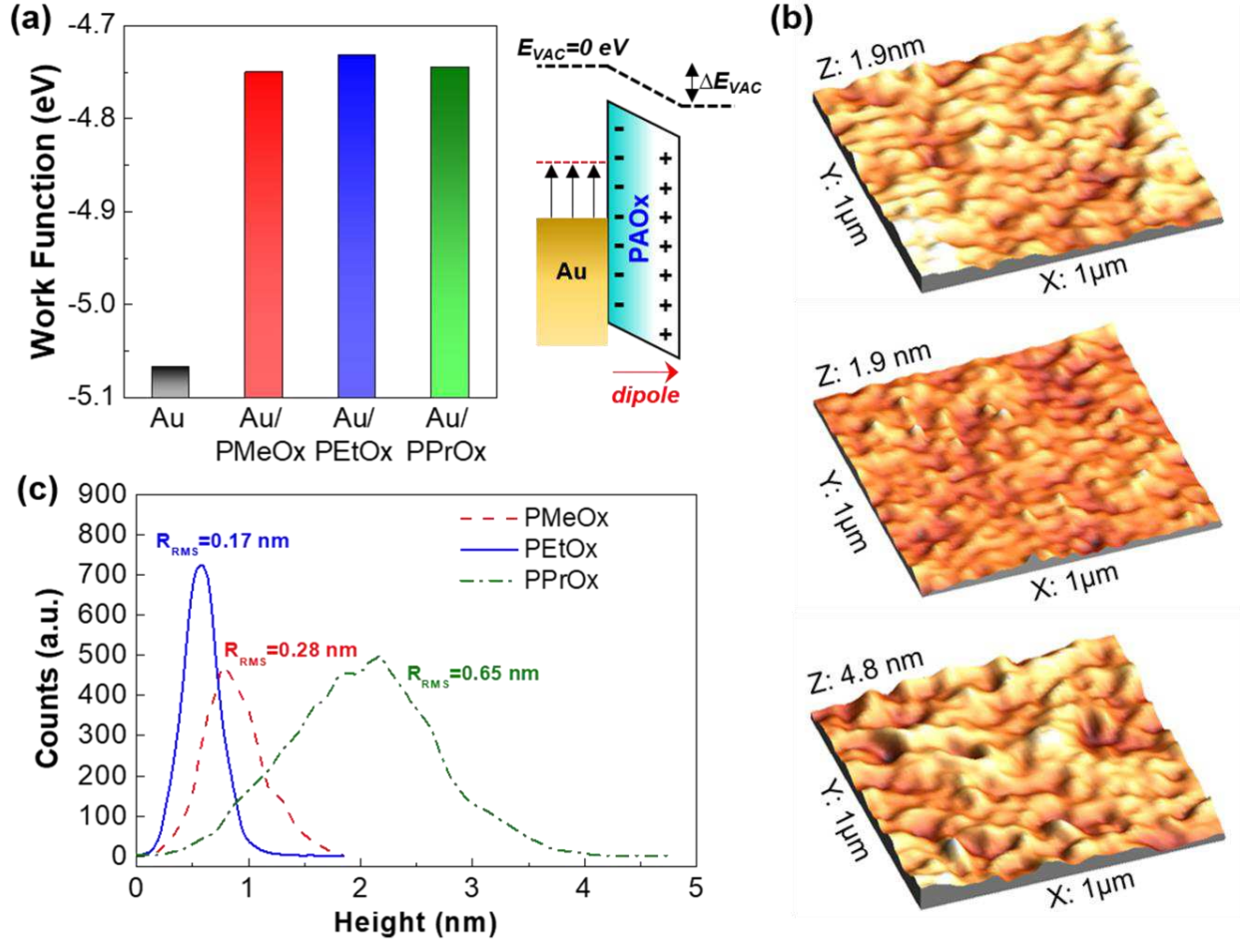

FIG. 4 (a) Work function values measured by Kelvin probe for Au and for Au with PMeOx, PEtOx, and PPrOx interlayers coated on top and work function shift caused by the PAOx interlayer dipole. (b) 3D-height mode AFM images and (c) height distributions for PMeOx (red dashed line), PEtOx (blue solid line), and PPrOx (green dot-dashed line) layers on glass substrates. 
(a)

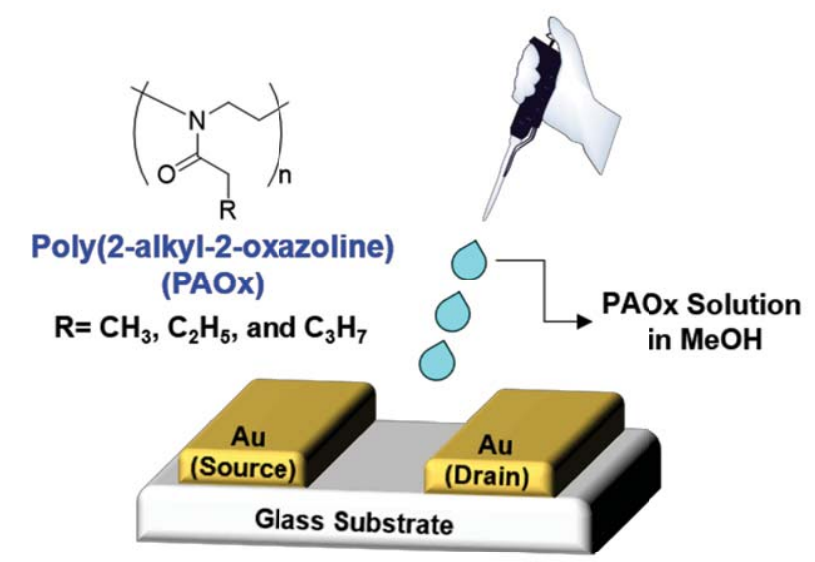

(b)

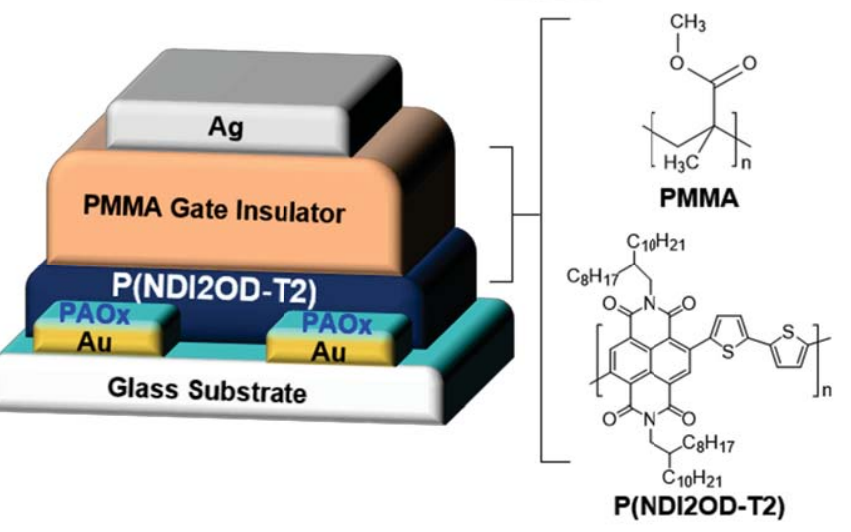


(a)

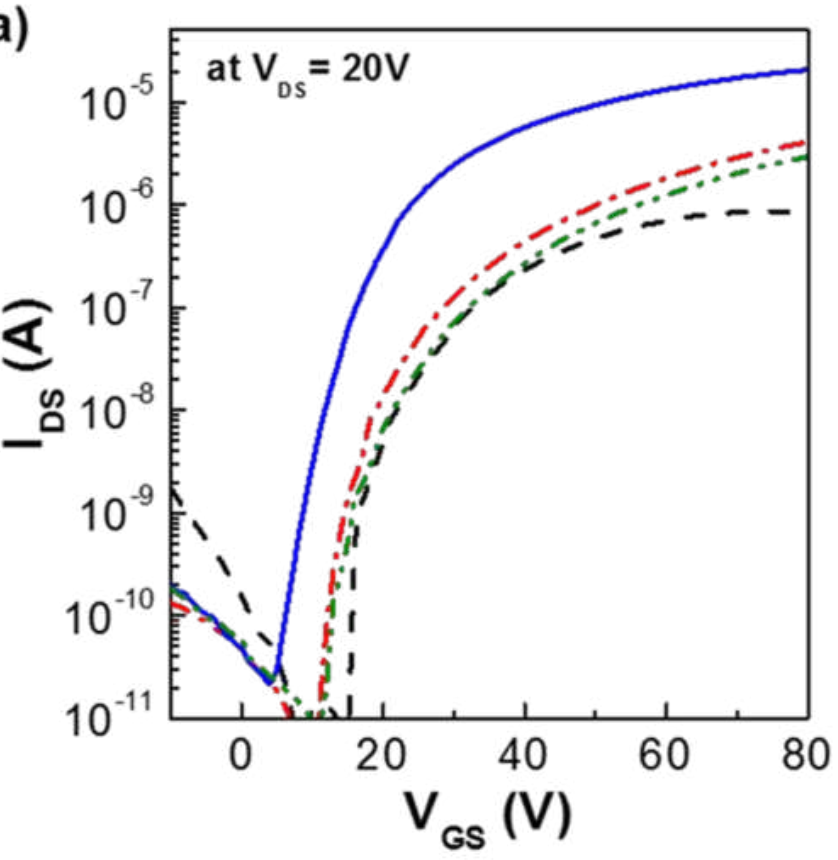

(b)

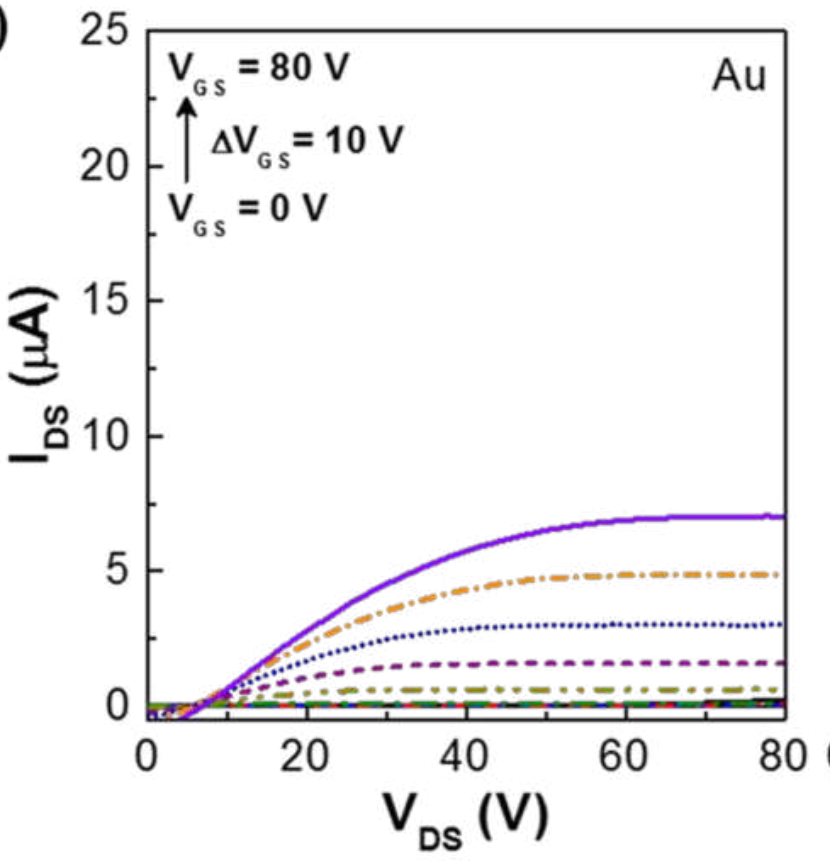

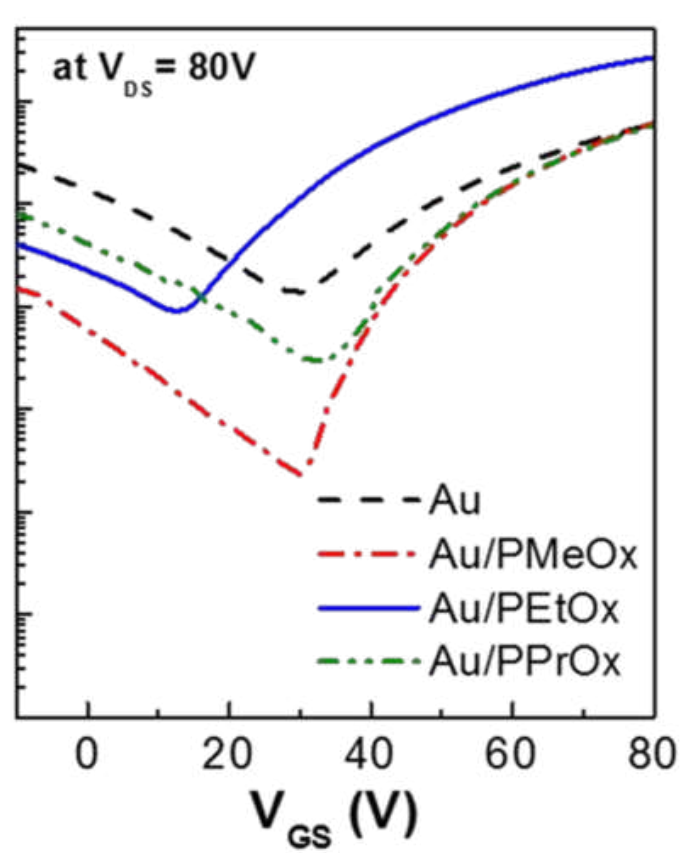

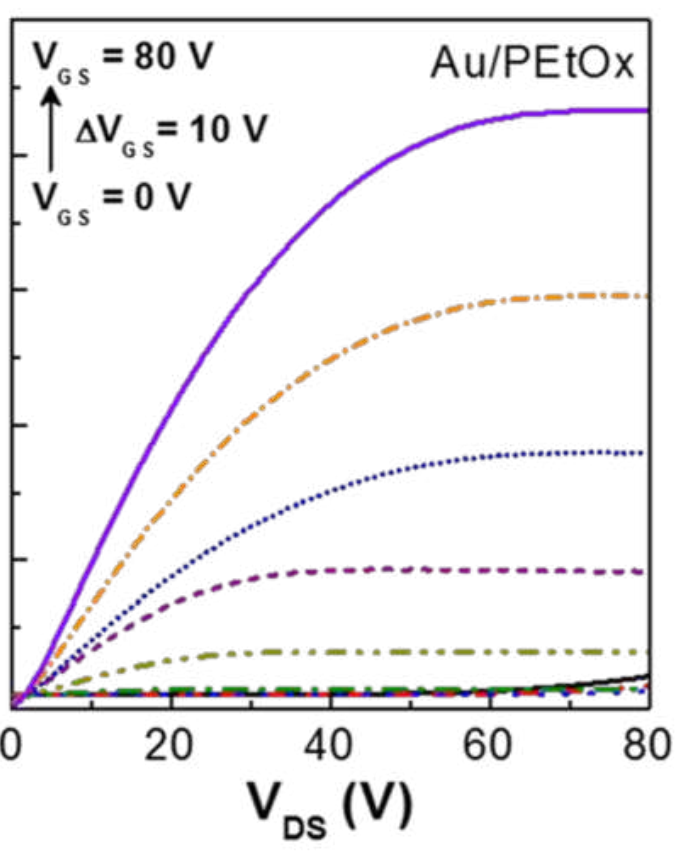


(a)

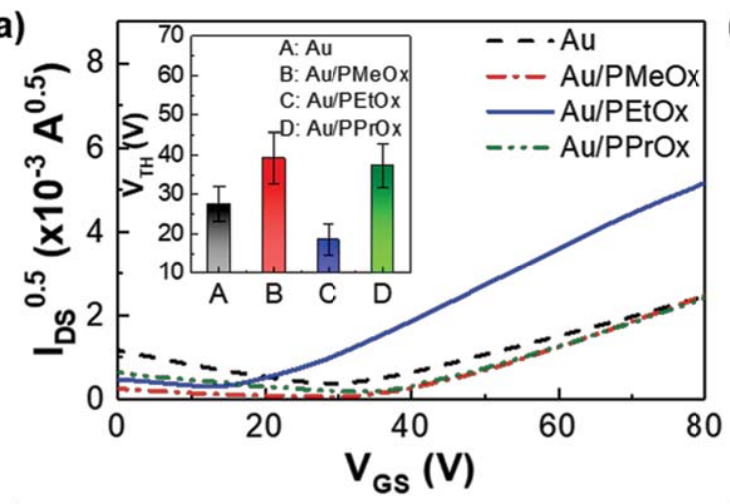

(c)

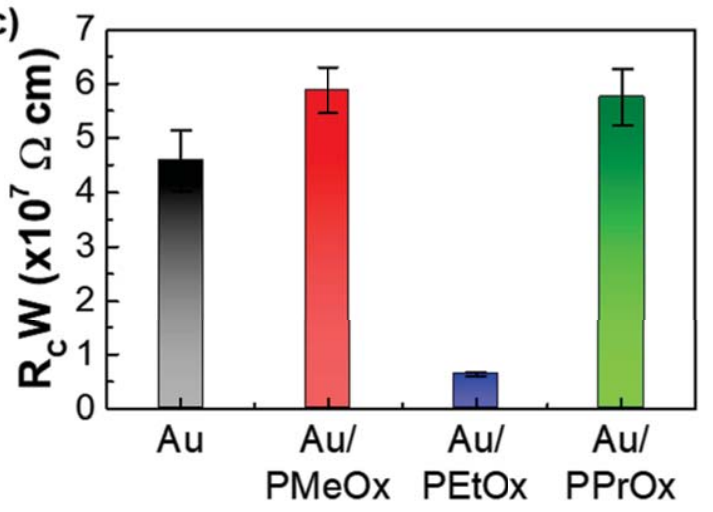

(b)

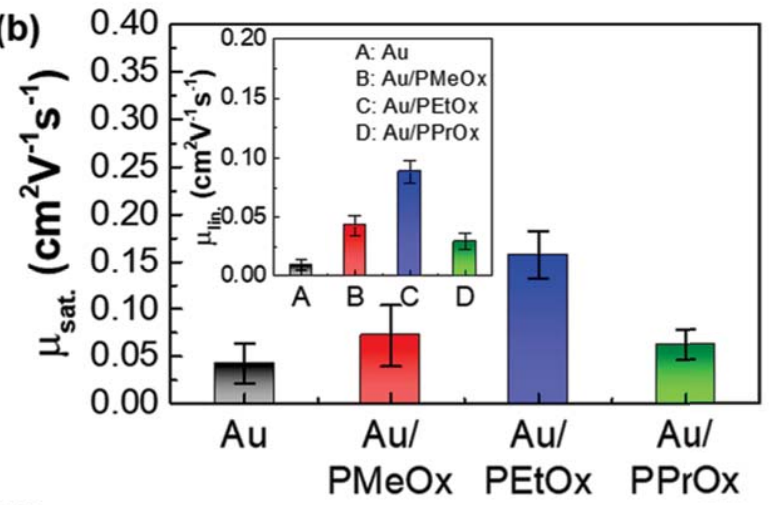

(d)

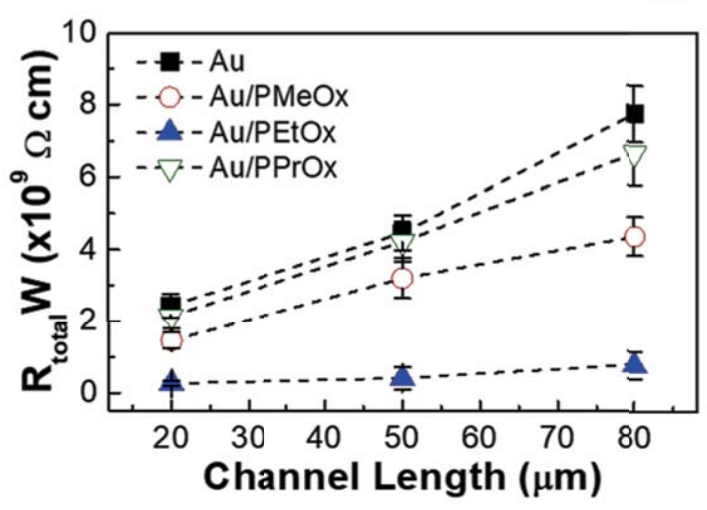



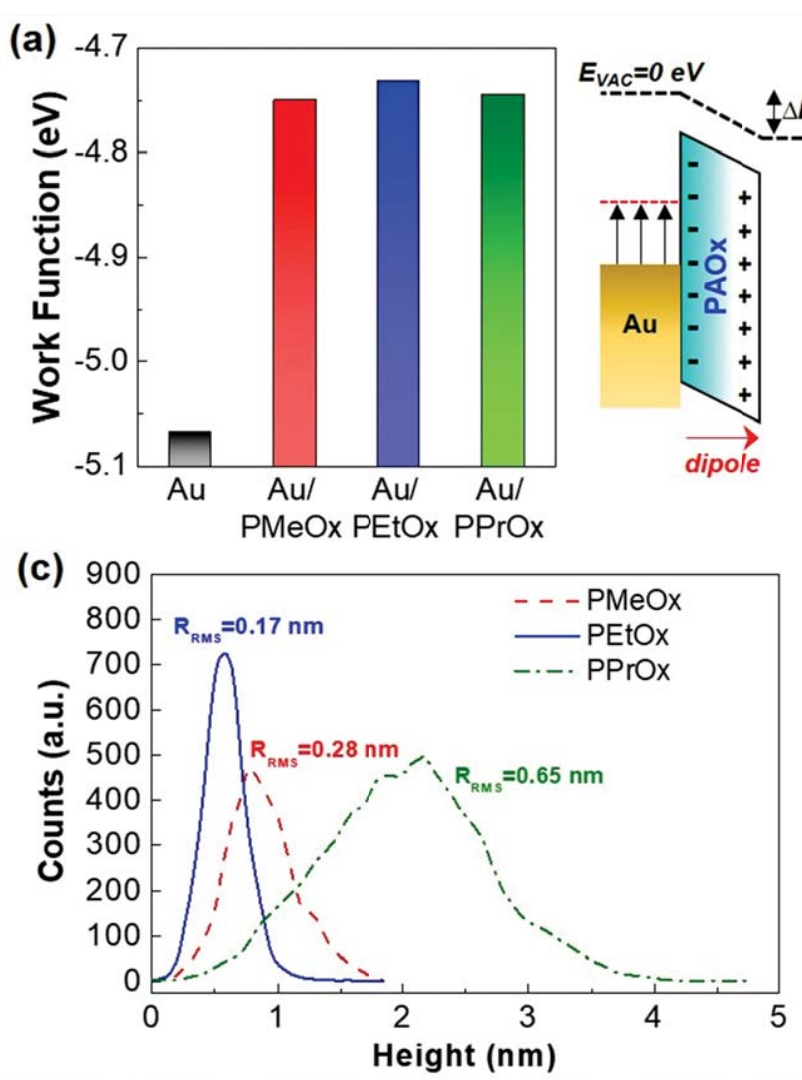

(b)
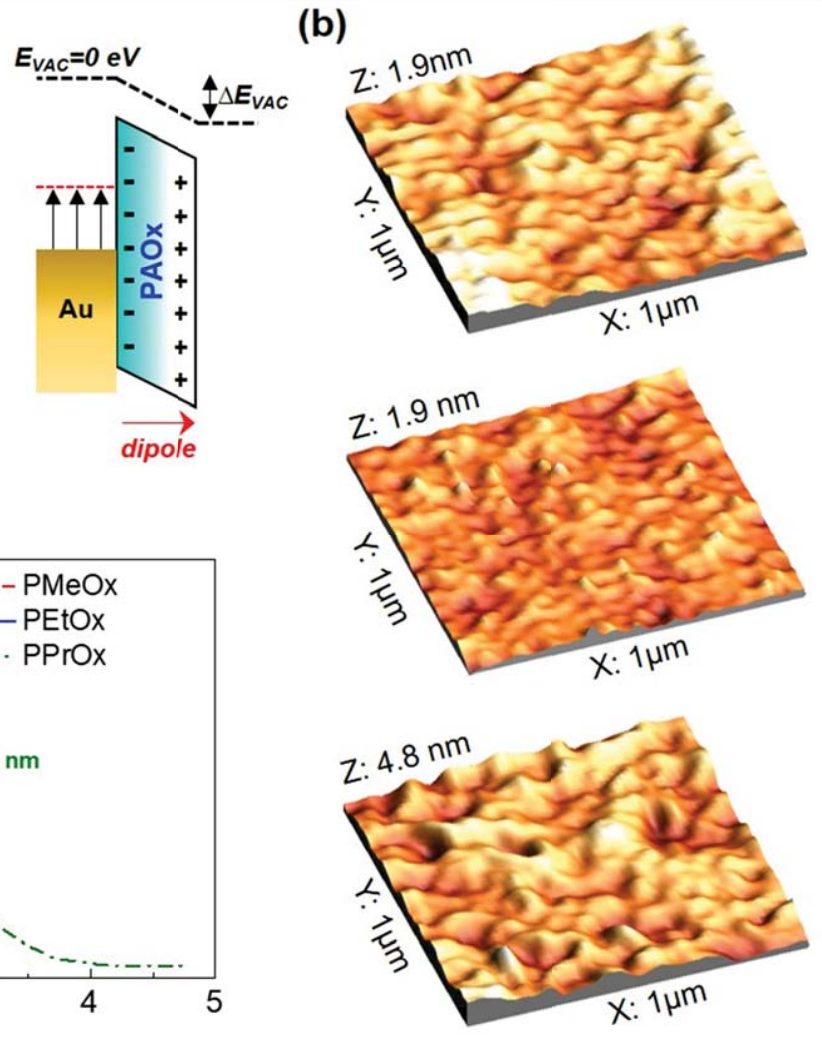